\title{
ERGODIC AND MIXING PROPERTIES OF MEASURES ON LOCALLY COMPACT ABELIAN GROUPS
}

\author{
THOMAS RAMSEY AND YITZHAK WEIT
}

\begin{abstract}
We provide new proofs of the theorems of Choquet and Deny and of Foguel concerning iterates of convolutions of a measure on a locally compact abelian group.
\end{abstract}

Let $G$ be a locally compact abelian group, $\Gamma$ its dual group and $m$ its Haar measure. $\mu$ denotes a bounded, complex, Borel measure on $G$ with Fourier-Stieltjes transform $\hat{\mu}$ on $\Gamma$.

The ergodic and mixing properties of probability measures $\mu$ were characterized by Choquet and Deny [1] and by Foguel [2]. In this note we provide new proofs which are transparent from the point of view of harmonic analysis. In the concluding remark, the proof for the theorem of Choquet and Deny is extended naturally to compact nonabelian groups. Let $I_{0}$ denote $\left\{f \in L_{1}(G): \hat{f}(e)=0\right\}$, where $e$ is the identity of $\Gamma$ and $\mu^{n}$ is the $n$-times convolution of $\mu$.

THEOREM 1. Let $\mu$ be a bounded, complex Borel measure on $G$ such that $\left\|\mu^{n}\right\| \leq$ $c$ for all $n$. Then $\lim _{n \rightarrow \infty}\left\|\mu^{n} * f\right\|_{1}=0$ for $f \in I_{0}$ if and only if $|\hat{\mu}(\gamma)|<1$ for all $\gamma \in \Gamma \backslash\{e\}$.

REMARK 1. If $\mu$ is a probability measure, then the condition that $|\hat{\mu}(\gamma)|<1$ for $\gamma \in \Gamma \backslash\{e\}$ is equivalent to Foguel's condition that the support of $\mu$ is not contained in any set of the form $\{x \in G:(x, \gamma)=c\}$ for any constant $c$ and for $\gamma \in \Gamma \backslash\{e\}$.

PROOF. Let $I=\left\{f \in I_{0}:\left\|\mu^{n} * f\right\|_{1} \rightarrow 0\right.$ as $\left.n \rightarrow \infty\right\}$.

$I$ is clearly a closed ideal in $L_{1}(G)$. Because points of $\Gamma$ obey synthesis, to prove $I=I_{0}$ it suffices to prove that $I_{0}$ is the only regular maximal ideal of $L_{1}(G)$ that contains $I[3,39.29]$. Stated more simply, for each $\gamma \in \Gamma \backslash\{e\}$ it suffices to produce $f \in I$ such that $\hat{f}(\gamma) \neq 0$.

Let $\gamma \in \Gamma \backslash\{e\}$. Choose $K$, a compact neighborhood of $\gamma$ excluding $e$. Choose $h \in L_{1}(G)$ such that $0 \leq \hat{h} \leq 1,\left.\hat{h}\right|_{K}=1$, and the support of $\hat{h}$ is a compact set excluding $e$. Let $f$ be any function in $L_{1}(G)$ such that $\hat{f}$ is supported in $K$ and $\hat{f}(\gamma) \neq 0$. Then

$$
\left\|\mu^{n} * f\right\|_{1}=\left\|\mu^{n} * h^{n} * f\right\|_{1} \leq\|f\|_{1}\left\|(\mu * h)^{n}\right\|_{1} .
$$

By the spectral radius theorem

$$
\lim _{n \rightarrow \infty}\left\|(\mu * h)^{n}\right\|_{1}^{(1 / n)}=\operatorname{Sup}\{|\hat{\mu}(\gamma) \hat{h}(\gamma)|: \gamma \in \Gamma\} .
$$

This last is less than 1 and therefore $\lim _{n \rightarrow \infty}\left\|(\mu * h)^{n}\right\|_{1}=0$. By (1) this implies that $f \in I$, which completes the proof.

Received by the editors November 23, 1983.

1980 Mathematics Subject Classification. Primary 43A25, $60 \mathrm{~J} 15$.

(C) 1984 American Mathematical Society $0002-9939 / 84 \$ 1.00+\$ .25$ per pag ${ }^{\circ}$ 
For the ergodic properties we have the following simple proof of the theorem of Choquet and Deny.

THEOREM 2. Let $\mu$ be a bounded, complex, Borel measure on $G$. Then the following are equivalent:

(i) for $f \in L_{\infty}(G), \mu * f=f$ implies $f=$ constant.

(ii) $\hat{\mu}(\gamma) \neq 1$ for $\gamma \in \Gamma \backslash\{e\}$.

REMARK 2. For $\mu$ a probability measure, the condition $\hat{\mu}(\gamma) \neq 1$ for $\gamma \in \Gamma \backslash\{e\}$ is equivalent to the fact that the support of $\mu$ generates a dense subgroup of $G$; i.e. that $\mu$ is adapted.

ProOF. Let $W=\left\{f \in L_{\infty}(G): \mu * f=f\right\}$.

$W$ is a $w^{*}$-closed, translation invariant subspace of $L_{\infty}(G)$. For $x \in \Gamma$ we have $\mu * x=\hat{\mu}(x) x=x$. Therefore, $x \in \Gamma \cap W$ if and only if $\hat{\mu}(x)=1$.

Suppose that $W$ consists only of constant functions ( $W=\mathbf{C e}$ or $W=\{0\}$ ). Then there is at most one continuous character in $W$, the identity $e$. For all other elements of $\Gamma$ we have $\hat{\mu}(\gamma) \neq 1$.

Conversely, suppose that $\widehat{\mu}(\gamma) \neq 1$ for $\gamma \neq e, \gamma \in \Gamma$. Then $W$ can contain at most one continuous character, namely $e$. If $e$ is not in $W$ we may apply an $L_{\infty}(G)$-form of Wiener's Theorem to conclude that $W=\{0\}[\mathbf{3}, 40.7]$. If $e$ is in $W$ we must use again the fact that $e$, as a point of $\Gamma$, obeys synthesis. By $[3,40.23(\mathrm{~b})]$, since $W \cap \Gamma$ is exactly $\{e\}, W=\mathbf{C} e$.

REMARK 3. Using the Peter-Weyl Theorem for compact (nonabelian) groups $G$, one may simply characterize minimal one-sided translation-invariant, $w^{*}$-closed subspaces of $L_{\infty}(G)$. By modifying the proof of Theorem 2 one can show that adapted probability measures are ergodic [4].

\section{REFERENCES}

1. G. Choquet and J. Deny, Sur l'équation de convolution $\mu=\mu * \sigma$, C. R. Acad. Sci. Paris 250 (1960), 799-801.

2. S. R. Foguel, On iterates of convolutions, Proc. Amer. Math. Soc. 47 (1975), 368-370.

3. E. Hewitt and K. Ross, Abstract harmonic analysis, Vol. II, Springer-Verlag, New York, 1970.

4. K. Ito and Y. Kawada, On the probability distribution on a compact group. I, Proc. Phys.-Math. Soc. Japan 22 (1940), 226-278.

Department of Mathematics, University of HaWAil at ManoA, Manoa, HaWAiI Department of Mathematics, Ben Gurion University, Beersheva, Israel

Current address (Thomas Ramsey): Mitre Corporation, $\mathrm{C}^{3} \mathrm{I}$ Division, 1820 Dolley Madison Boulevard, McLean, Virginia 22102

Current address (Yitzhak Weit): Department of Mathematics, University of Connecticut, Storrs, Connecticut 06268 